\title{
Delayed Branch Retinal Artery Occlusion and Partial Oculomotor Nerve Palsy Following Coiling of a Giant Intracavernous Carotid \\ Artery Aneurysm
}

Keywords: Branch retinal artery occlusion; Oculomotor nerve palsy; Coil embolization; Giant carotid cavernous aneurysm; Visual field; Sc otoma; Ptosis

\begin{abstract}
Coil embolization is a non-invasive and effective method of treating intracranial a neurysm s not amendable to surgical clipping by inducing thrombus formation. However, ophthalmic complic ations and cranial nerve injury are rarely encountered following the procedure. We report a case of inferior branch retinal artery occlusion and partial oculomotor nerve palsy of the right eye seven days after coil embolization and balloon occlusion of a giant intracavemous right intemal carotid artery a neurysm in an 18 year old healthy Caucasian man. After three months, there was resolution of the oculomotomene palsy, but superior arcuatescotoma remained unchanged. Baseline ophthalmic examinations and discussion of potential ophthalmic risks with patients prior to the interventional aneurysm coiling procedures are recommended.
\end{abstract}

\section{Abbreviations}

ICA: Internal Carotid Artery; BRAO: Branch Retinal Artery Occlusion; CRAO: Central Retinal Artery Occlusion; CTA Computed Tomography Angiography; MRA: Magnetic Resonance Angiography; MRI: Magnetic Resonance Imaging

\section{Introduction}

Over the past twenty-three years, coil embolization has emerged as an effective and minimally-invasive neuro-radiological treatment of intracranial aneurysms. Selective occlusion of the unruptured aneurysms is achieved by deployment of detachable coils through an endovascular route to induce thrombus formation within the aneurysm sac. Not only does coil embolization allow for the treatment of intracranial aneurysms that are unamenable to surgical clipping but also allows patients to avoid risks associated with the neurosurgical approach with $4 \%$ morbidity and $1 \%$ mortality even in elective cases [1]. However, coil embolization is not without its own set of potential complications, and ophthalmic complications are rare adverse events that may result from embolic events or ischemia secondary to direct compression and neurovascular compromise [2-4]. We herein report a case of delayed inferior branch retinal artery occlusion and transient partial oculomotor nerve palsy of the right eye after coil embolization and balloon occlusion of a giant intracavernous right internal carotid artery aneurysm.

\section{Case Report}

An 18 year-old healthy Caucasian college student presented to the emergency department with a five day history of worsening right

\section{Journal of}

\section{Ocular Biology}

\section{Sarah Bonnet ${ }^{1}$, Brian Ellis ${ }^{2}$, Jeffrey S Carpenter ${ }^{3}$,} John Nguyen ${ }^{2 *}$

${ }^{1}$ School of Medicine, West Virginia University, Morgantown, $W V$, USA

${ }^{2}$ Department of Ophthalmology, West Virginia University, Morgantown, WV, USA

${ }^{3}$ Department of Radiology, West Virginia University, Morgantown, WV, USA

*Address for Correspondence

John Nguyen, MD, 1 Stadium Dr., PO Box 9193, Morgantown, WV 26506-9193,USA, Tel: 304-598-6925; E-mail: nguyenj@ wvuhealthcare.com

Submission: 01 August 2013

Accepted: 23 August 2013

Published: 27 August 2013

Reviewed \& Approved by: Dr. Bjørn Nicolaissen

Department of Ophthalmology

University of Oslo, Norway

temporal headaches that were not relieved with over the counter non-steroidal anti-inflammatory drugs. A partially-thrombosed giant aneurysm, measuring $4.7 \times 4.1 \times 3.5 \mathrm{~cm}$, involving the cavernous portion of the right internal carotid artery (ICA) was seen on neuroimaging (Figures 1 and 2). A balloon occlusion test of the right ICA with cerebral arteriography demonstrated that the patient would be able to tolerate pa rent vessel sacrifice. Coil embolization of the giant right cavernous ICA aneurysm with proximal vessel sacrifice was then performed using platinum detachable coils. Upon completion of the coil embolization and balloon occlusion, angiography revealed coils throughout the aneurysm and the distal petrous segment of the right internal carotid artery with appropriate retrograde filling into the right supraclinoid internal carotid artery to supply the right ophthalmic artery. Angiographic studies obtained after balloon deflation revealed no significant flow into the aneurysm (Figure 3).

At the conclusion of the procedure, the ophthalmology service was consulted for conjunctivalchemosis and proptosis of the right

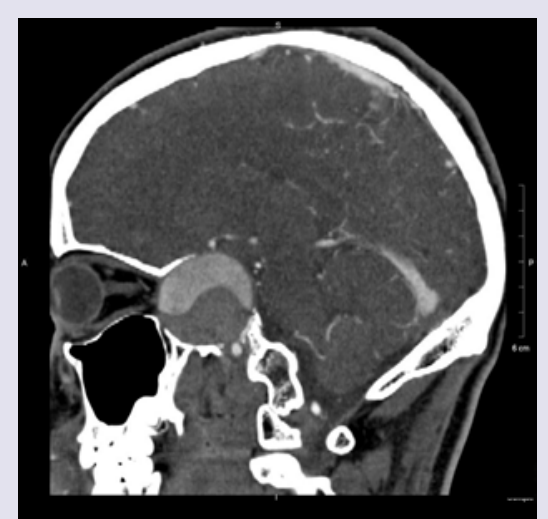

Figure 1: A computed tomography angiography study of the brain revealed a partially-thrombosed giant aneurysm involving the cavernous portion of the right internal carotid artery. 
Citation: Bonnet S, Ellis B, Carpenter JS, Nguyen J. Delayed Branch Retinal Artery Occlusion and Partial Oculomotor Nerve Palsy Following Coiling of a Giant Intracavernous Carotid Artery Aneurysm. J Ocular Biol. 2013;1(1): 4.

eye. His past ocular history was significant for myopia. The initial exam revealed a moderately firm right orbit without pulsation, and intraocular pressures of $16 \mathrm{mmHg}$ OD and $14 \mathrm{mmHg}$ OS. There was mild restriction of right eye motility in all gazes. No ptosis was noted. The visual acuity was 20/20 in each eye. The pupils were round and reactive without a relative afferent pupillary defect. The anterior segment exam and the dilated fundus exam were unremarkable. No cranial nerve deficits were seen. Repeat angiography revealed full patency of the intracranial arteries. No significant filling into the aneurysm was appreciated and no carotid cavernous fistulae or venous filling abnormalities were present. The patient was given $4 \mathrm{mg}$ of intravenous dexamethasone every six hours and placed on heparin, and he had tapering of corticosteroid with resolution of chemosis and proptosis during 4 days of hospitalization. The patient was discharged on a continuation of prednisone taper, aspirin, as well as warfarin for prophylaxis of cavernous sinus thrombosis and had a therapeutic INR of 2.5 .

Two days later, the patient returned to the emergency department with blurry vision, eye pain, and dilation of the right pupil. The visual acuity was 20/20 in each eye. Anisocoria was present with pupillary diameter of $7 \mathrm{~mm}$ OD and $4 \mathrm{~mm}$ OS in the dark and 6 $\mathrm{mm}$ OD and $3 \mathrm{~mm}$ OS in the light. No relative pupillary defect was observed. There was mild restriction of right eye motility in upgaze, adduction and depression. The right upper eyelid was mildly ptotic with margin-reflex distance of $1.5 \mathrm{~mm}$ OD and $4 \mathrm{~mm}$ OS, and the orbit was not tense to retropulsion. The intraocular pressures were $13 \mathrm{mmHg}$ OD and $15 \mathrm{mmHg}$ OS. The dilated fundus exam showed retinal whitening surrounding inferior branch retinal artery of the right eye. No embolus was noted (Figure 4). A Humphrey visual field exam showed a superior arcuatescotoma and superior nasal step in the right eye (Figure 5). A diagnosis of partial oculomotor nerve palsy and inferior branch retinal artery occlusion was made. Laboratory workup for primary thrombotic and autoimmune phenomena was negative. His INR was found to be supratherapeutic at 3.7 and was adjusted. In addition, he was switched from oral prednisone to 500 $\mathrm{mg}$ intravenous solumedrol every twelve hours. Magnetic resonance angiography (MRA) revealed extensive thrombosis of the previous right ICA aneurysm with some continued filling. Magnetic resonance imaging (MRI) of the brain revealed no hemorrhage or infarction. He was discharged with Coumadin, aspirin, and a corticosteroid taper.

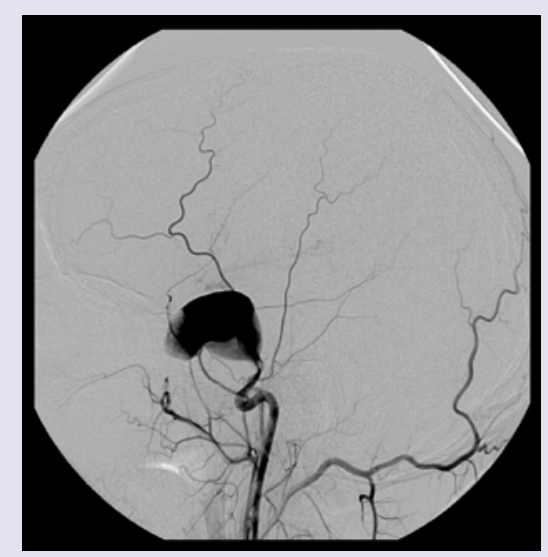

Figure 2: A cerebral angiography image that demonstrated a partially thrombosed giant intracavernous right internal carotid artery aneurysm.

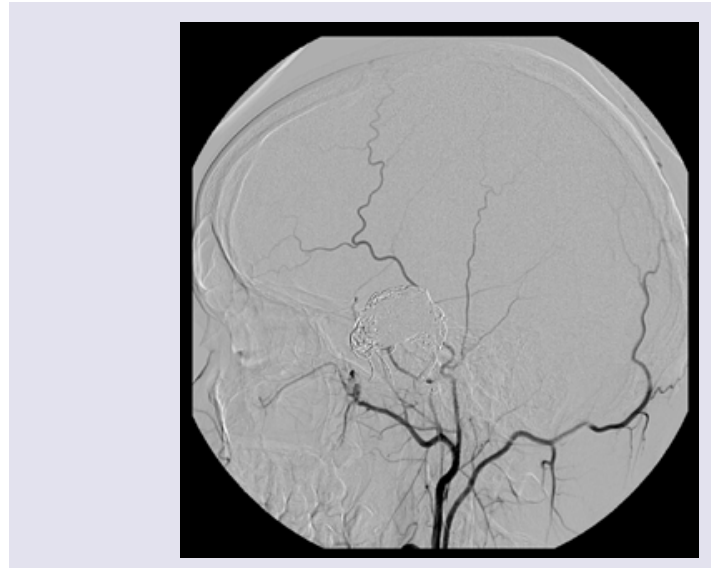

Figure 3: Cerebral angiography after coil embolization of the giant right cavernous internal carotid artery aneurysm with proximal vessel sacrifice revealed no significant flow into the aneurysm.

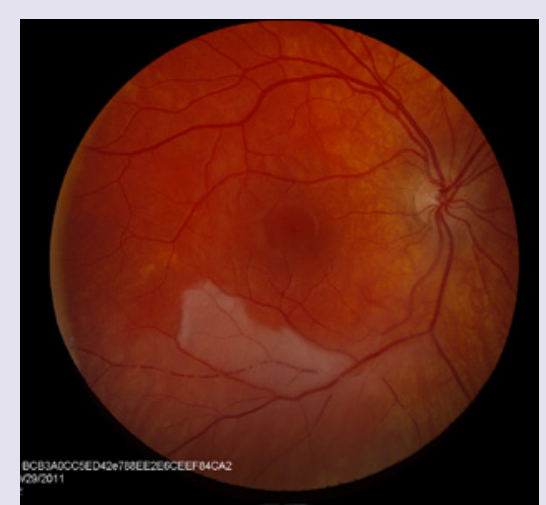

Figure 4: Fundus photo of the right eye showed retinal whitening surrounding inferior branch retinal artery of the right eye.

At three months follow up, there was resolution of partial oculomotor nerve palsy but no improvement of the superior nasal scotoma of the right eye. Cerebral angiography 3 months after coil embolization revealed complete occlusion of the right internal carotid artery and no anterograde flow through the coil mass. There was a small amount of filling without evidence of coil compaction into the anteromedial portion of the aneurysm.

\section{Discussion}

New or worsening ophthalmic complications such as retinal artery occlusion and cranial nerve palsy are uncommon complications after coiling but have been reported in the literature. While cerebrovascular ischemia secondary to thromboembolism is the most common neurological adverse outcome with reported rate of $1 \%$ to $17 \%$, there have been only one report of branch retinal artery occlusion (BRAO) and two reports of central retinal artery occlusion subsequent to coiling of intracranial carotid aneurysms [4-6]. BRAO has also been seen in stenting of carotid artery stenosis secondary to embolic phenomenon [7].

Large aneurysm diameter and the presence of prolapsed loops of coil are thromboembolic risk factors and associated with an increased risk for cerebrovascular ischemia in 193 patients who had Guglielmi coil [3]. In Castillo et al. report, a 13 year old girl developed right 
Citation: Bonnet S, Ellis B, Carpenter JS, Nguyen J. Delayed Branch Retinal Artery Occlusion and Partial Oculomotor Nerve Palsy Following Coiling of a Giant Intracavernous Carotid Artery Aneurysm. J Ocular Biol. 2013;1(1): 4.

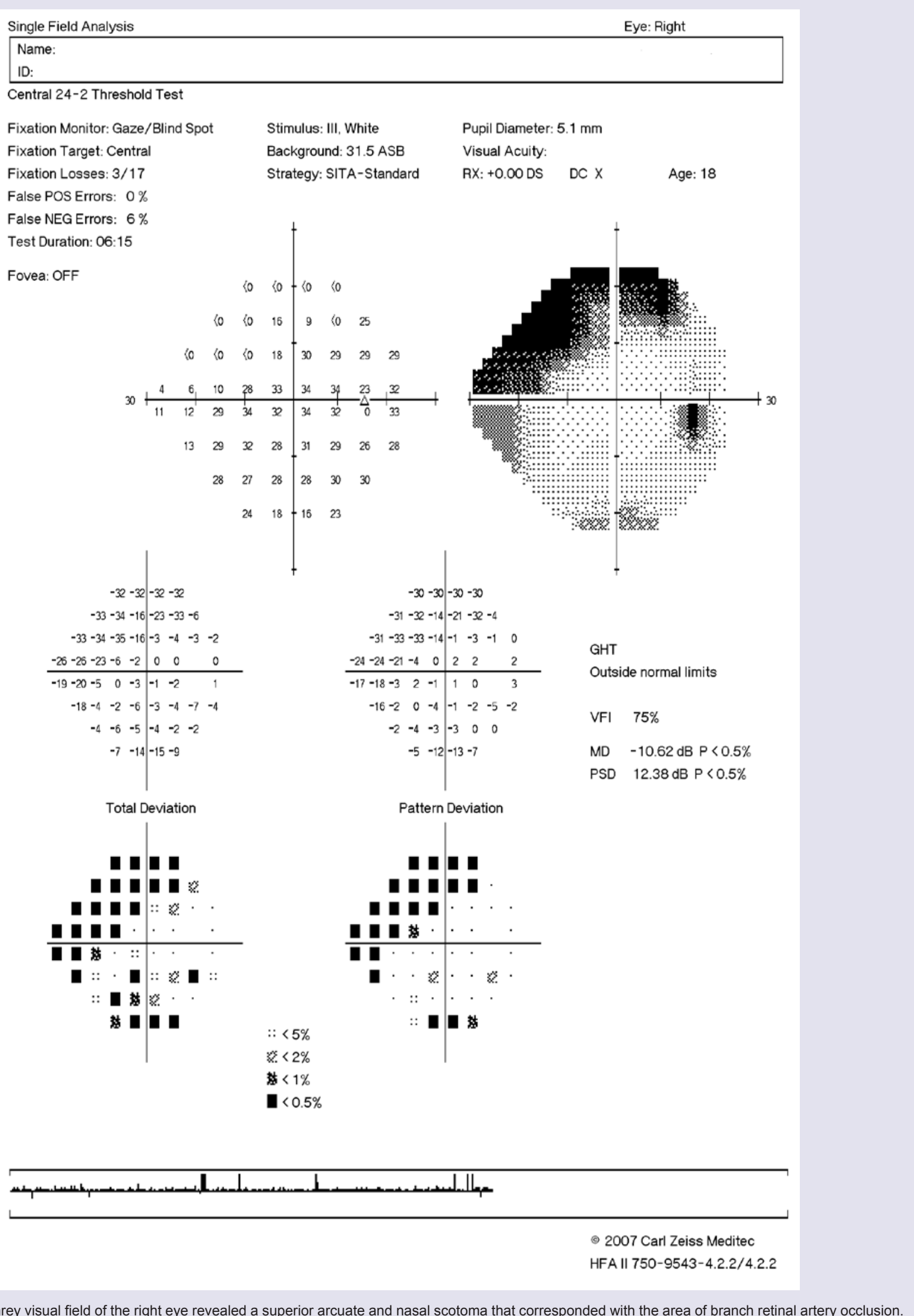

Figure 5: Humphrey visual field of the right eye revealed a superior arcuate and nasal scotoma that corresponded with the area of branch retinal artery occlusion. 
Citation: Bonnet S, Ellis B, Carpenter JS, Nguyen J. Delayed Branch Retinal Artery Occlusion and Partial Oculomotor Nerve Palsy Following Coiling of a Giant Intracavernous Carotid Artery Aneurysm. J Ocular Biol. 2013;1(1): 4.

superior branch retinal artery occlusion 2 days following coiling of an $8.6 \mathrm{~mm}$ aneurysm with Guigleilmi coil, and there was coil protrusion into the parent artery [1]. Our patient is also of young age but has a much larger aneurysm, a risk factor for thromboembolic event. In addition, BRAO developed a week after the procedure in our case despite being on anticoagulation. Central retinal artery occlusion (CRAO), on the other hand, was described in a case of perforation of a $4 \mathrm{~mm}$ aneurysm while coiling with subsequent withholding of heparin and in a different case of platinum coiling of a $5 \times 5 \mathrm{~mm}$ leaking aneurysm $[5,6]$. CRAO developed a few hours after the procedure in the former case and after 10 minutes in the latter case. In both cases, the patient's vision was hand motion, and only one patient had full visual recovery to 20/20. CRAO have not been described in a delayed setting. BRAO causes partial visual field loss and may not be as symptomatic early on, so it is critical to assess for visual changes after coiling for early detection. In addition, treated aneurysms may have thromboembolic potential for up to 2 months, so patients should be informed of this risk, to monitor for visual changes, and to seek urgent ophthalmic care if such event occurs [3].

Oculomotor nerve palsy by itself or in combination with other cranial nerve palsies or Horner's syndrome is a common presenting symptom of carotid cavernous aneurysms, and diplopia is the most common manifestation [8]. However, new onset oculomotor nerve palsy is uncommon. Several cases have been documented in the literature with varying times of onset and duration. Development of perioperative oculomotor nerve palsy can arise from Gugleilmi coiling-related hemorrhage [9]. Transient oculomotor nerve palsy, identified after the coiling of wide-neck basilar tip aneurysms, typically resolved within 24 hours [10]. In addition, $\mathrm{Xu}$ et al reports three patients with new onset oculomotor nerve palsies presenting more than 1 year after coiling and associated with new or recurrent flow in their aneurysm without changes in aneurysm sizes [11]. Transient worsening of cranial nerve dysfunction and followed by improvement or resolution within weeks to months after coil embolization of intracranial aneurysms has been previously described and attributed to the acute stage of aneurysm thrombosis and temporary inflammatory enlargement and mass effect [1] Oculomotor palsy in our patient was associated with pain and found seven days after the procedure, and we hypothesized that transient inflammatory enlargement of the aneurysm and mass effect maybe contributing factors leading to an ischemic oculomotor palsy.

Lastly, the acute conjunctivalchemosis and proptosis immediately following coiling is uncommonly reported. Our patient had significant aneurysm induced osseous remodeling, especially at the orbital apex (Figure 1) for which fluctuations of intracranial pressure would be readily transmitted to the orbit and adnexal structures. The acute stage of aneurysm thrombosis may have induced a transient mass effect to cause orbital venous outflow obstruction and increased intra-orbital pressure leading to orbital congestion, proptosis, and conjunctivalchemosis. The patient had resolution of these findings as the inflammatory enlargement was remedied with corticosteroid.

In conclusion, coil embolization of intracavernous aneurysms carries the risk of ophthalmic events such as branch retinal artery occlusion and partial oculomotor nerve palsy. We strongly recommend a baseline ophthalmic examination and discussion of ophthalmic risks with patients prior to undergoing such interventions. In addition, follow up ophthalmic assessment and cranial nerve exams after coil embolization may help in early detection of these potentially devastating complications.

\section{References}

1. Castillo B Jr, De Alba F, Thornton J, DeBrun G, Pulido J (2000) Retinal artery occlusion following coil embolization of carotid-ophthalmic aneurysms. Arch Ophthalmol 118: 851-852

2. Malisch TW, Guglielmi G, Viñuela F, Duckwiler G, Gobin YP, et al. (1998) Unruptured aneurysms presenting with mass effect symptoms: response to endosaccular treatment with Guglielmi detachable coils. Part I. Symptoms of cranial nerve dysfunction. J Neurosurg 89: 956-961.

3. Turner RD, Byrne JV, Kelly ME, Mitsos AP, Gonugunta, et al. (2008) Delayed visual deficits and monocular blindness after endovascular treatment of large and giant paraophthalmic aneurysms. Neurosurgery 63: 469-474.

4. Derdeyn CP, Cross DT 3rd, Moran CJ, Brown GW, Pilgram TK, et al. (2002) Postprocedure ischemic events after treatment of intracranial aneurysms with Guglielmi detachable coils. J Neurosurg 96: 837-843.

5. Ascaso FJ, Cristobal JA (1999) Partial retinal artery occlusion after coil embolization of an intracerebral aneurysm. Eur J Ophthalmol 9: 142-144.

6. Castillo BV Jr, Khan AM, Gieser R, Shownkeen H (2005) Purtscherlike retinopathy and Horner's syndrome following coil embolization of an intracavernous carotid artery aneurysm. Graefes Arch Clin Exp Ophthalmo 243: $60-62$

7. Lee SJ, Kim SY, Kim SD (2009) Two cases of branch retinal arterial occlusion after carotid artery stenting in the carotid stenosis. Korean J Ophthalmol 23 53-56.

8. Stiebel-Kalish $\mathrm{H}$, Kalish $\mathrm{Y}$, Bar-On $\mathrm{RH}$, Setton A, Niimi $\mathrm{Y}$, et al. (2005) Presentation, natural history, and management of carotid cavernous aneurysms. Neurosurgery 57: 850-857.

9. Kwon BJ, Han MH, Oh CW, Kim KH, Chang KH (2003) Procedure-related haemorrhage in embolisation of intracranial aneurysms with Guglielmi detachable coils. Neuroradiology 45: 562-569.

10. Thorell WE, Chow MM, Woo HH, Masaryk TJ, Rasmussen PA (2005) $Y$-configured dual intracranial stent-assisted coil embolization for the treatment of wide-necked basilar tip aneurysms. Neurosurgery 56: 10351040.

11. Xu DS, Hurley MC, Batjer HH, Bendok, BR (2010) Delayed cranial nerve palsy after coiling of carotid cavernous sinus aneurysms: case report. Neurosurgery 66: E1215-E1216. 